\title{
Guide pour l'évaluation des méthodes d'analyses de routine
}

\author{
par \\ R. GRAPPIN \\ I.N.R.A., Station Expérimentale Laitière - 39800 Poligny
}

A côté des méthodes chimiques ou bactériologiques traditionnelles, souvent longues à exécuter et demandant du personnel qualifié, les laboratoires utilisent de plus en plus des méthodes d'analyse de routine leur permettant d'effectuer rapidement un nombre très élevé d'analyses.

Une méthode d'analyse de routine doit être une méthode simple, rapide, peu onéreuse et pouvant si possible être exécutée par du personnel peu spécialisé. Généralement, c'est une méthode plus ou moins automatisée, l'automatisation pouvant être réalisée, soit à partir d'une méthode déjà connue et éprouvée, soit le plus souvent à partir de méthodes physiques facilement automatisables (spectrophotométrie, réfractométrie, etc.) reposant la mesure de caractéristiques biochimiques ou de propriétés particulières du produit à analyser. Dans ce cas, le principe de la méthode de routine est différent de celui des méthodes existantes.

Lorsque l'on a affaire à un appareil automatique d'analyse, il est parfois difficile de faire une distinction précise entre la méthode d'analyse proprement dite et l'appareil basé sur cette méthode. Aussi, les appareils font-ils rarement l'objet de normes d'analyses, au sens stricte du terme, mais plus simplement l'objet de guides ou de "normes d'utilisation ».

Lorsqu'une nouvelle méthode a été mise au point, qu'il s'agisse d'une méthode dont le principe diffère de celui de la méthode de référence ou qu'il s'agisse simplement de l'automatisation d'une méthode de référence, il faut toujours procéder à une étude préalable de la "valeur » de cette nouvelle méthode.

Evaluer une méthode d'analyse de routine comporte l'étude de trois aspects distincts : la validité du principe sur lequel est basé la méthode, sa valeur technique et économique et sa valeur analytique.

- La validité du principe d'une méthode peut se résumer ainsi : la méthode nous renseigne-t-elle exactement sur ce que nous voulons 
savoir de la qualité ou de la composition du produit analysé ? Est-elle valable pour atteindre le but fixé ? Par exemple, pour apprécier la qualité bactériologique du lait, on ne choisira pas obligatoirement la même méthode selon qu'on utilise ce critère comme méthode de contrôle de l'hygiène à la production, ou comme méthode de contrôle de la qualité technologique du lait. L'étude de la validité procède plus d'une réflexion technique et scientifique que d'une méthode expérimentale.

- La valeur technique et économique de la méthode comporte l'étude de caractéristiques bien connues que nous ne faisons que mentionner ici : la fiabilité dans le cas d'un appareil, la souplesse d'utilisation, les facilités de travail, les risques divers, le coût par analyse, les investissements, etc.

- Troisième aspect, la valeur analytique ou précision de la méthode. C'est essentiellement à ce niveau que se situe le travail de l'expérimentateur chargé d'étudier une méthode d'analyse.

Dans ce document, nous donnerons tout d'abord un certain nombre de définitions se rapportant à la précision d'une méthode car de nombreuses confusions sont faites dans l'utilisation des termes tels que fidélité, répétabilité, précision, exactitude, etc. d'une méthode. Nous exposerons ensuite les méthodes statistiques qu'il convient d'utiliser pour évaluer les différents paramètres définissant la précision. Pour cela, nous nous reporterons essentiellement, lorsqu'ils existent, aux normes ou documents édités par l'AFNOR (1) ou l'ISO ; dans le cas contraire, soit nous nous référerons à des travaux en cours effectués par des groupes spécialisés de l'ISO, soit nous proposerons des définitions et méthodes d'évaluations qui nous semblent actuellement les mieux adaptées.

Il nous a paru également utile de donner la traduction anglaise des principaux termes utilisés dans l'étude de la précision d'une méthode d'analyse.

\section{PRECISION}

\subsection{Définition}

- Précision (2) "Soit $m$ une mesure effectuée à l'aide d'une méthode d'analyse et $\mu$ la valeur vraie de la grandeur à mesurer, la précision de la méthode est définie par la différence $m-\mu »$.

(1) - Norme NF X 06-041 : fidélité d'une méthode d'essai, guide pour les essais interlaboratoires.

- J. LaURent (1971) : livre blanc sur la normalisation des méthodes d'ana. lyse chimique (A.F.N.O.R.).

(2) En anglais : accuracy. 


\subsection{Estimation}

La précision est une notion globale de la qualité ou de la valeur analytique d'une méthode. Elle comprend plusieurs caractéristiques principales qui sont la fidélité, la justesse, la sensibilité. Une méthode sera d'autant plus précise, c'est-à-dire la différence $m$ - $\mu$ sera d'autant plus faible, que sa fidélité, sa justesse et sa sensibilité seront plus élevées. Etant donné son caractère essentiellement qualitatif, la précision, en tant que telle, ne possède en général pas de valeur bien définie. On limitera donc son emploi au jugement d'une méthode, lequel jugement devra se baser sur les valeurs des autres caractéristiques mesurables (fidélité, justesse et sensibilité) que nous allons étudier.

Nous soulignons dès à présent que nous ne prenons en considération que les méthodes d'analyses normalisées ou pouvant faire l'objet de " normes d'utilisation ", dont les résultats s'expriment de manière quantitative et appliquées à des échantillons de produit n'ayant subi aucune modification susceptible d'influencer le résultat d'analyse.

\section{LA FIDELITE}

La fidélité d'une méthode est certainement la caractéristique la mieux définie. Elle fait l'objet de normes AFNOR et ISO, pour sa définition et son estimation (NF X 06-041, ISO/DIS 3534 (3)) et de projets de normes étudiés par des groupes spécialisés de l'ISO (ISO/ TC $69 /$ GTB).

Après avoir donné différentes définitions, nous examinerons les méthodes d'estimation de la fidélité.

\subsection{Définitions}

- Fidélité (4) (norme ISO/DIS 3534) : " étroitesse de l'accord entre les résultats obtenus en appliquant le procédé expérimental à plusieurs reprises dans des conditions déterminées.

Le procédé est d'autant plus fidèle que la partie aléatoIre des erreurs expérimentales qui affectent les résultats est moindre 》.

Les différents résultats d'analyse d'un même produit obtenus à l'aide d'une méthode donnée peuvent être réalisés soit dans des conditions identiques (même laboratoire, même opérateur, laps de temps réduit), soit dans des conditions différentes. Suivant le cas,

(3) Nouvelle norme internaticnale concernant la fidélité, remplaçant la recommandation ISO/R 645 .

(4) En anglais : precision. 
la variabilité des résultats risque d'être différente. La fidélité de la méthode sera donc définie par deux critères : la répétabilité lorsque les conditions expérimentales sont identiques et la reproductibilité lorsque les conditions expérimentales sont différentes.

- RéPÉTABIltté (5) (Norme ISO/DIS 3534) : "étroitesse de l'accord entre les résultats successifs obtenus avec la même méthode sur une matière identique soumis à l'essai et dans les mêmes conditions".

Dans les mêmes conditions, signifie que les déterminations ont été faites avec le même opérateur, le même équipement, dans le même laboratoire et dans un intervalle de temps très court.

- ReProductibilité (6) (Norme ISO/DIS 3534) : " étroitesse de l'accord entre les résultats individuels obtenus avec la même méthode sur une matière identique soumise à l'essai et dans des conditions différentes ».

Par conditions différentes, on entend essentiellement le cas de déterminations faites par des opérateurs différents dans des laboratoires différents.

Entre les conditions extrêmes définies pour la répétabilité d'une part et pour la reproductibilité d'autre part, on peut trouver toutes les situations intermédiaires: opérateurs différents travaillant dans le même laboratoire, déterminations faites avec le même appareil mais à des époques différentes, etc. On peut être ainsi amené à définir et à calculer une reproductibilité entre opérateurs ou entre appareils, une reproductibilité journalière, etc. Les méthodes de calcul se rapportant à ces différentes reproductibilités sont calquées sur les méthodes plus générales que nous allons donner.

Les définitions de la répétabilité et de la reproductibilité données ci-dessus, peuvent être utilement complétées par des définitions quantitatives plus concrètes :

- La répétabilité $(r)$ et la reproductibilité $(R)$ sont définies chacune par la valeur en dessous de laquelle est située, avec une probabilité spécifiée, la valeur absolue de la différence entre deux résultats individuels $x_{1}$ et $x_{2}$ obtenus soit dans des conditions de répétabilité, soit dans des conditions de reproductibilité. En l'absence d'indication, la probabilité est de 95 p. 100.

Soit : $P\left(\left|x_{1}-x_{2}\right| \leqslant r\right)=0,95$ et $P\left(\left|x_{1}-x_{2}\right| \leqslant R\right)=0,95$.

En d'autres termes, $r$ et $R$ représentent dans chaque cas, l'écart arithmétique entre deux déterminations ayant une probabilité de 95 p. 100 de ne pas être dépassée.

(5) En anglais : repeatability.

(6) En anglais : reproducibility. 


\subsection{Estimation de la répétabilité et de la reproductibilité}

\subsubsection{Modèle mathématique}

La fidélité (répétabilité et reproductibilité) d'une méthode d'analyse doit être estimée à partir d'un ou plusieurs essais inter-laboratoires (7). Afin de donner les expressions mathématiques de la répétabilité et de la reproduztibilité nous indiquerons les grandes lignes du modèle qu'il convient d'utiliser pour effectuer un tel essai.

Chaque résultat d'analyse (pour un échantillon donné) est la somme de trois composants : $\mathrm{y}=\mathrm{m}+\mathrm{b}+\mathrm{e}$.

$\mathrm{m}$ est la moyenne générale des résultats fournis par tous les laboratoires, elle est considérée ici comme étant la valeur vraie de la variable.

b représente la différence moyenne de chaque laboratoire par rapport à $\mathrm{m}$. Cette composante est distribuée normalement autour de $\mathrm{m}$ avec une variance $\sigma_{\mathrm{L}}^{2}$ appelée variance entre laboratoires. On admet que les laboratoires ayant participé à l'essai représentent un échantillon de laboratoires provenant d'une population beaucoup plus grande.

e représente l'erreur aléatoire attachée à chaque résultat, elle est distribuée normalement avec une moyenne égale à 0 et une variance $\sigma_{r}^{2}$. Cette variance représente la variance intra-laboratoire obtenue au cours de l'essai ou variance de répétabilité.

\subsubsection{Méthode statistique}

Les essais inter-laboratoires comportent l'analyse par p laboratoires (au moins 8) de plusieurs échantillons situés à q niveaux de la grandeur étudiée, par la méthode d'analyse dont on veut évaluer la fidélité. Chaque échantillon fait l'objet dans chaque laboratoire de $n$ répétitions.

Le cas le plus simple consiste à définir la fidélité par niveau en effectuant une analyse de variance à une voie à partir de laquelle on calcule les deux variances $\sigma_{\mathrm{r}}^{2}$ et $\sigma_{\mathrm{L}}^{2}$ (tab. $1 \mathrm{~A}$ ).

A partir de ces données, on obtient aisément la variance de reproductibilité $\sigma_{\mathrm{R}}^{2}$ qui est la somme de la variance de répétabilité et de la variance entre laboratoires : $\sigma_{k}^{2}=\sigma_{r}^{2}+\sigma_{L}^{2}$.

Avant de tirer toute conclusion, il faut s'assurer à l'aide de tests appropriés que les erreurs intra-laboratoires et entre laboratoires sont distribuées normalement. On utilise pour cela le test de Cochran pour vérifier l'homogénéité des variances intra-laboratoires et le test de Dixon pour éliminer éventuellement les laboratoires ayant des moyennes anormales.

(7) En anglais : interlaboratory trial. 


\section{TABLEAU 1}

Estimation des variances de répétabilité et de reproductibilité d'une méthode d'analyse

A : Analyse par niveau

\begin{tabular}{l|c|c|c|c}
\hline $\begin{array}{c}\text { Origine } \\
\text { de la } \\
\text { variation }\end{array}$ & $\begin{array}{c}\text { Somme des } \\
\text { carrés des } \\
\text { écarts }\end{array}$ & $\begin{array}{c}\text { Degré } \\
\text { de } \\
\text { liberté }\end{array}$ & Carré moyen & $\begin{array}{c}\text { Estimation } \\
\text { des } \\
\text { variances }\end{array}$ \\
\hline Laboratoire & $\operatorname{SCE}_{\mathrm{L}}$ & $\mathrm{p}-1$ & $\mathrm{SCE}_{\mathrm{L}} /(\mathrm{p}-1)$ & $\sigma^{2}+\mathrm{n}_{\mathrm{L}}^{2}$ \\
Répétition & $\mathrm{SCE}_{\mathrm{r}}$ & $\mathrm{p}(\mathrm{n}-1)$ & $\mathrm{SCE}_{\mathrm{r}} / \mathrm{p}(\mathrm{n}-1)$ & $\sigma_{\mathrm{r}}$ \\
\hline
\end{tabular}

B : Analyse globale

\begin{tabular}{|c|c|c|c|c|}
\hline Laboratcire & $\mathrm{SCE}_{\mathrm{L}}$ & p-1 & $\mathrm{SCE}_{\mathrm{L}} /(\mathrm{p}-1)$ & $n q \sigma^{2}{ }_{L}+n \sigma_{L x N}^{2}+\sigma_{r}^{2}$ \\
\hline $\begin{array}{l}\text { Interaction } \\
\text { laboratoire } \mathrm{x} \text { niveau }\end{array}$ & $\mathrm{SCE}_{\mathrm{LxN}}$ & $(\mathrm{p}-1)(\mathrm{q}-\mathrm{l})$ & $\mathrm{SCE}_{\mathrm{LxN}} /(\mathrm{p}-1)(\mathrm{q}-1)$ & $\mathrm{n} \sigma_{\mathrm{LxN}}^{2}+\sigma_{r}^{2}$ \\
\hline Répétition & $\mathrm{SCE}_{\mathrm{r}}$ & $\mathrm{pq}(\mathrm{n}-\mathrm{l})$ & $\mathrm{SCE}_{\mathrm{r}} / \mathrm{pq}(\mathrm{n}-\mathrm{l})$ & $\sigma_{r}^{2}$ \\
\hline
\end{tabular}

Tableau d'analyse de la variance, par niveau ou globale, des résultats d'analyse obtenus par $\mathrm{p}$ laboratoires, pour $\mathrm{q}$ niveaux de la grandeur à mesurer et $\mathrm{n}$ répétitions.

En reprenant les définitions énoncées ci-dessus, on aura :

- répétabilité $: \mathrm{r}=2,77 . \sigma_{\mathrm{r}}$,

- reproductibilité $: \mathrm{R}=2,77 . \sigma_{\mathrm{R}}$.

Le coefficient 2,77 est tiré de la loi de distribution des valeurs extrêmes (ou étendues) d'une population et varie en fonction du nombre d'observations par échantillon (il est égal à 2,77 pour $n=2$ ).

Dans le cas où on fait une analyse globale pour q niveaux, on effectue une analyse de variance à deux voies (modèle croisé, aléatoire) ; le carré moyen entre répétitions sera toujours une estimation correcte de $\sigma_{r}^{2}$. Pour calculer $\sigma_{\mathrm{R}}^{2}$ il faut introduire la variance $\sigma_{\mathrm{L}, \mathrm{N}}^{2}$ de l'interaction laboratoire $\mathrm{x}$ niveau (tab. 1 B). On aura : $\sigma_{\mathrm{B}}^{2}=\sigma_{\mathrm{L}}^{2}+$ $\sigma_{\mathrm{L} \times \mathrm{N}}^{2}+\sigma_{\mathrm{r}}^{2}$.

Dans tous les cas et en particulier pour les méthodes d'analyses bactériologiques (numération bactérienne, comptage des cellules), il 
faut s'assurer que $\sigma^{2}$ et $\sigma_{\mathrm{R}}^{2}$ sont indépendants du niveau, sinon on effectuera une transformation des données pour uniformiser les variances. Pour cela, il convient d'établir exactement la relation cntre les variances $\sigma_{r}^{2}$ et $\sigma_{R}^{2}$, calculées pour chaque niveau et les moyennes par niveau. Si la variance est proportionnelle à la moyenne de la variable, on doit effectuer une transformation racine carrée, si c'est l'écart-type qui est proportionnel, on effectue une transformation logarithmique des données.

Rappelons à ce propos que s'il est possible après une transformation logarithmique de revenir aux données d'origine en exprimant $r$ et $R$ en valeur relative (c'est-à-dire en p. 100 par rapport à la valeur la moins élevée), il n'est théoriquement pas possible de faire cette conversion dans le cas d'une transformation racine carrée. Il s'ensuit que si la transformation racine carrée est très utile pour effectuer des tests d'hypothèse, elle est en revanche fort peu pratique pour faire une estimation. En effet, l'expression de $r$ et $\mathrm{R}$ en racine carrée des données d'origine est difficilement utilisable par l'analyste. Dans ce cas, il semble préférable d'exprimer la fidélité indépendamment pour chaque niveau.

\subsection{Application aux méthodes et appareils d'analyse de routine}

L'application des principes énoncés ci-dessus aux méthodes et appareils d'analyses de routine peut se faire en tenant compte des réserves suivantes :

10 Il est souvent difficile d'évaluer la répétabilité d'un nouvel appareil automatique en suivant le schéma indiqué car on ne dispose en général que d'un nombre très réduit d'appareil (parfois un seul) pour faire les essais.

Dans ce cas, on estimera la répétabilité à partir de $\mathrm{n}$ déterminations $(n \geqslant 2)$ faites sur $p$ échantillons différents (au lieu de p laboratoires) pour chaque niveau. La variance de répétabilité (8) sera calculée de la même manière par analyse de la variance (tab. $1 \mathrm{~A}$ ).

$2^{\circ}$ Dans le cas des appareils d'analyse qui sont calibrés par l'utilisateur (voir chapitre 3), la reproductibilité est étroitement liée à l'exactitude de calibrage des différents appareils. Il ne sera donc possible de donner la reproductibilité pour un type d'appareil que s'il existe des échantillons étalons permettant de standardiser le calibrage de tous les appareils mis en œuvre dans l'essai interlaboratoire. Dans le cas contraire, la valeur de reproductibilité trouvée ne sera pas une caractéristique propre de ce type d'appareil mais plutôt une caractéristique des calibrages actuels des différents appareils.

(8) En anglais, pour $\mathrm{n}=2$, souvent appelé " variance between duplicate ". 


\section{JUSTESSE}

Actuellement, aucune norme précise n'existant pour la justesse d'une méthode d'analyse, la plupart des définitions et méthodes que nous donnons ne doivent être considérées que comme des propositions et outils de travail susceptibles de pouvoir être modifiés et améliorés.

\subsection{Définitions}

3.1.1. Justesse (9) (ISO/DIS 3534) : " étroitesse de l'accord entre la vraie valeur de la grandeur à mesurer et le résultat moyen qui serait obtenu en appliquant le procédé expérimental un grand nombre de fois. Le procédé est d'autant plus juste que la PARTIE SYSTÉMATIQUE des erreurs expérimentales qui affectent les résultats est moindre ".

L'évaluation de la justesse d'une méthode d'analyse revient à comparer la valeur vraie de la grandeur à mesurer, donnée en général par une méthode de référence pour laquelle il aura été admis, par convention, qu'elle donne la valeur vraie, avec la valeur moyenne de plusieurs déterminations obtenues par la méthode de routine (de façon à diminuer les erreurs de fidélité). La notion de justesse s'applique donc à toutes les méthodes d'analyses, autres que les méthodes de référence et en particulier aux méthodes de routine.

Les méthodes de routine étant très souvent des méthodes physiques d'analyse (spectrophotométrie par exemple), il faut relier les mesures données par l'appareil (absorbance dans l'exemple de la spectrophotométrie) aux résultats donnés par la méthode de référence et ceci à l'aide d'un calibrage (10) approprié. Le calibrage est donc un facteur important dans la justesse d'une méthode, mais il n'est pas le seul, car une partie de l'erreur systématique entre la méthode de référence et la méthode de routine tient aux différences de principe ou de mode opératoire existant entre les deux méthodes. On distinguera donc dans la justesse deux notions différentes :

- l'exactitude de calibrage,

- la précision par rapport à la méthode de référence ou précision d'estimation.

Ces deux notions sont illustrées par la figure 1. L'ellipse de la figure représente la dispersion des poinıs de coordonnées $\overline{\mathrm{x}}_{2}, \mathrm{y}_{2}$ d'une population d'échantillons d'un produit donné, analysés par la méthode de référence et par la méthode de routine que l'on veut tester.

3.1.2. ExACTITUDE DE CALIBRAGE (11) : "étroitesse de l'accord entre la droite observée et la droite théorique de calibrage de l'appareil. Le calibrage sera exact si les deux droites sont confondues ».

(9) En anglais : accuracy of the mean.

(10) On utilise également le terme d'étalonnage défini comme la détermination expérimentale des erreurs de justesse.

(11) En anglais : calibration accuracy. 


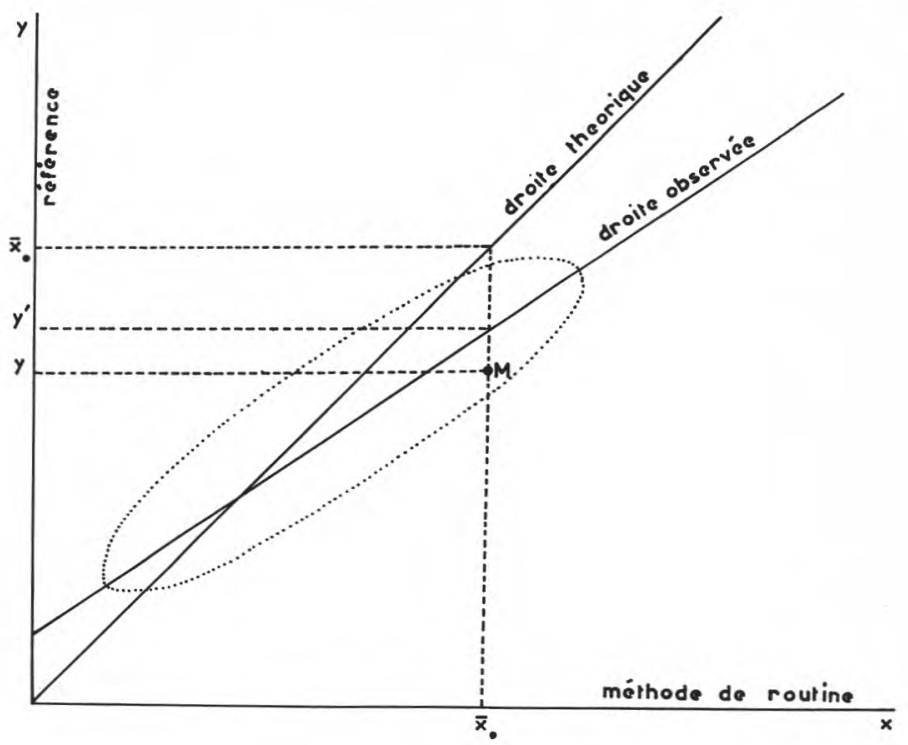

fig. 1

Décomposition des facteurs intervenant dans la justesse d'une méthode de routine

$\overline{\mathrm{x}}_{0}$ : moyenne de plusieurs déterminations avec la méthode de routine pour l'échantillon $\mathrm{M}$.

y : valeur vraie de la grandeur à mesurer donnée par la méthode de référence.

$\mathrm{y}^{\prime}$ : valeur moyenne donnée par la méthode de référence pour tous les échantillons correspondant à une donnée $\overline{\mathbf{x}}_{0}$ de la méthode de routine.

$$
\bar{x}_{0}-\mathrm{y}
$$

justesse

$$
=\quad \begin{gathered}
\left(\mathrm{y}^{\prime}-\mathrm{y}\right) \\
\text { précision } \\
\text { d'estimation }
\end{gathered}
$$

$$
\begin{aligned}
& +\quad\left(\bar{x}_{0}^{\prime}-y^{\prime}\right) \\
& \text { exactitude } \\
& \text { d'étalonnage }
\end{aligned}
$$

— "La droite observée de calibrage (12) est définie par l'équation de la droite de régression $y=b x+a$, calculée à partir des résultats obtenus avec un grand nombre d'échantillons ou avec des échantillons étalons analysés par la méthode de référence $(y)$ et par l'appareil $(x) »$.

- "La droite théorique de calibrage (13) est définie par l'équation $y=b^{\prime} x+a^{\prime}$ de la droite permettant d'avoir à tous les niveaux

(12 En anglais : observed calibration line.

(13) En anglais : theoritical calibration line. 
envisagés des résultats donnés par la méthode de routine qui soient en moyenne égaux aux résultats de la méthode de référence ".

Soulignons que dans le cas particulier du calibrage d'une méthode indirecte de routine, on cherche à estimer la valeur vraie de la grandeur, donnée par la méthode de référence, à partir de la mesure effectuée par la méthode de routine. Par conséquent, on construira le graphique et on effectuera les calculs en considérant la méthode de routine comme méthode indépendante $(\mathrm{x})$ et la méthode de référence comme méthode dépendante (y).

Pratiquement, on peut donner pour l'exactitude de calibrage, la définition suivante :

"Le calibrage d'une méthode d'analyse sera exact si la valeur du coefficient de régression (14) et la valeur de l'ordonnée à l'origine (15) de la droite observée ne sont pas statistiquement différentes (au seuil de 0,05) des valeurs correspondantes de la droite théorique de calibrage».

Dans le cas, très fréquent, d'un appareil donnant des mesures de concentration, la droite théorique de calibrage correspond à la droite ayant une pente de $1\left(b^{\prime}=1\right)$ et passant par l'origine $\left(a^{\prime}=0\right)$, (équation $\mathrm{y}=1 \cdot \mathrm{x}+0$ ).

3.1.3. PRÉCISION PAR RAPPORT A LA MÉTHODE DE RÉFÉRENCE OU PRÉCISION D'ESTIMATION (16): "Etroitesse de l'accord entre les moyennes d'un grand nombre de résultats obtenus par la méthode de référence et

\section{$T A B L E A U 2$}

Calcul de la précision d'estimation d'une méthode d'analyse de routine

\begin{tabular}{l|c|c|c|c}
\hline $\begin{array}{c}\text { Origine } \\
\text { de la } \\
\text { variation }\end{array}$ & $\begin{array}{c}\text { Somme } \\
\text { des } \\
\text { carrés }\end{array}$ & $\begin{array}{c}\text { Degré } \\
\text { de } \\
\text { liberté }\end{array}$ & Carré moyen & $\begin{array}{c}\text { Estimation } \\
\text { des } \\
\text { variances }\end{array}$ \\
\hline & $\mathrm{SCE}_{1}$ & 1 & $\mathrm{SCE}_{1}$ & \\
$\begin{array}{l}\text { Régression linéaire } \\
\begin{array}{l}\text { Ecarts par rapport } \\
\text { à la régression li- } \\
\text { néaire }\end{array}\end{array}$ & $\mathrm{SCE}_{y, x}$ & $\mathrm{n}-2$ & $\mathrm{SCE}_{y, x} / \mathrm{n}-2$ & $\sigma_{y, x}^{2}$ \\
\hline
\end{tabular}

Tableau d'analyse de la variance de la régression de y (méthode de référence) sur $\mathrm{x}$ (méthode de routine).

(14) En anglais : regression coefficient, slope.

(15) En anglais : level, intercept.

(16) En anglais : accuracy of estimate, ou souvent : accuracy. 
la méthode de routine sur un matériel identique, après élimination des déviations dues à un défaut éventuel de calibrage de la méthode de routine $"$

Pratiquement : " si $\bar{x}_{0}$ est la valeur moyenne donnée par une méthode de routine, la précision d'estimation de cette méthode est donnée par les limites $T_{1}$ et $T_{2}$ de l'intervalle statistique de dispersion à l'intérieur desquelles se situe avec une probabilité de 95 p.100 la vraie valeur y pour la valeur donnée $\bar{x}_{i}$. Soit : $P\left(T_{1}<y<T_{z}\right)=0,95$.

En d'autres termes, la précision d'estimation représente la précision avec laquelle la vraie valeur de la grandeur à mesurer est estimée par la méthode de routine dans 95 p. 100 des cas.

\subsection{Estimation de la justesse}

L'exactitude de calibrage ne constitue réellement une caractéristique d'un appareil d'analyse que dans la mesure où le calibrage ne dépend pas de l'opérateur.

En revanche, la précision d'estimation représente dans tous les cas, une mesure importante de la justesse et par conséquent de la valeur analytique d'une méthode indirecte et doit donc être clairement établie par les expérimentateurs.

\subsubsection{Méthodes de CALCul}

Pour déterminer l'exactitude de calibrage et la précision d'estimation, il faut analyser, au moins en double exemplaire, par la méthode de référence et par la méthode de routine approximativement calibrée, un nombre élevé d'échantillons du produit $(n \geqslant 40)$ choisis de façon à être situés régulièrement sur toute l'étendue habituellement observée de la variable.

3.2.1.1. Exactitude de calibrage : après avoir vérifié la linéarité de la relation entre les deux méthodes, on établit l'équation de régression observée $(\mathrm{y}=\mathrm{bx}+\mathrm{a})$ à partir des moyennes des résultats obtenus avec les deux méthodes d'analyse sur chaque échantillon.

L'exactitude de calibrage est vérifiée en comparant l'équation de la droite observée à l'équation de la droite théorique de calibrage. L'erreur provenant d'un calibrage inexact peut être soit une erreur systématique quel que soit le niveau (défaut d'ordonnée à l'origine), soit une erreur variable suivant le niveau (défaut de pente).

Lorsque la majorité des échantillons habituellement analysés se situe à un niveau proche de la moyenne, on peut se limiter à un test de comparaison des deux moyennes (de préférence à la comparaison des ordonnées à l'origine) et accorder une importance secondaire à l'exactitude de la pente.

3.2.1.2. Précision d'estimation : elle est calculée à partir de l'analyse de variance de la régression de y (méthode de référence) sur $\mathrm{x}$ 
(méthode de routine) d'où on tire la variance résiduelle $\sigma_{y_{1} \mathrm{x}}^{2}$ (tab. 2) et l'écart-type résiduel (17) $\sigma_{y, x}$. Suivant la définition donnée plus haut la précision d'estimation est de $\pm 1,96 . \sigma_{y_{x} x}$.

Lorsque, dans des conditions expérimentales bien définies, on obtient une valeur $\overline{\mathrm{x}}_{\text {o }}$ avec une méthode indirecte de routine correctement calibrée, la "vraie valeur " de la grandeur à mesurer se situe dans 95 p. 100 des cas entre $\bar{x}_{0}+1,96, \sigma_{y, x}$ et $\bar{x}_{0}-1,96 . \sigma_{y, x}$.

Signalons que la variance résiduelle calculée à partir de la régression est égale, à un degré de liberté près, à la valeur de la variance des écarts algébriques (18) entre les deux méthodes $\left(\sigma^{2}{ }_{y-x}\right)$ lorsque la pente de la droite de régression est voisine de 1 (dans le cas contraire, $\sigma_{y_{-x}}^{z}$ est supérieur à $\sigma_{y_{y} x}^{2}$ ).

Cette méthode de calcul plus simple peut éventuellement être employée.

On peut également donner la « précision relative » (19) de la méthode en calculant le coefficient de détermination (20) $r^{2}=$ $\sigma_{y}^{2}, x-\sigma_{y}^{2}$

$\sigma^{2}$ des y ayant servi de calcul.

La valeur $\mathrm{r}^{2}$ donne la proportion de la variance expliquée par la régression de $\mathrm{y}$ sur $\mathrm{x}$. Ce coefficient est surtout utile pour comparer entre elles les précisions d'estimation de plusieurs méthodes de routine appliquées à des variables ayant des intervalles de variation (ou étendue) différents.

Comme dans le cas de la fidelité, il faut s'assurer avant tout calcul que $\sigma_{y, s}^{2}$ est constant quel que soit le niveau. Dans le cas contraire, on opérera une transformation des données avec toutes les conséquences déjà évoquées plus haut. Soulignons que l'intérêt de déterminer la précision d'estimation est de fixer la part de l'erreur systématique qui provient de la différence entre les principes de la méthode de routine et de la méthode de référence.

Pour une méthode donnée, appliquée à un produit donné, la précision d'estimation est souvent fonction de la nature ou de l'origine des échantillons analysés. Ainsi, on constate par exemple, pour le dosage de la matière grasse du lait par spectrophotométrie infrarouge, que la précision d'estimation est moins bonne (écart-type résiduel plus élevé) dans le cas d'échantillons de laits individuels (provenant d'animaux différents) que dans le cas de laits de trou-

(17) En anglais : residual standard deviation, standard deviation from regression.

(18) En anglais : standard deviation of differences.

(19) En anglais : relative accuracy.

(20) En anglais : determination coefficient. 
peaux (provenant de troupeaux différents) simplement parce que la composition et les propriétés des laits individuels sont moins homogènes que celles des laits de troupeau (21). Aussi, lorsqu'on donne la précision d'estimation d'une méthode, est-il indispensable de spécifier quel type d'échantillons a été utilisé au cours des essais.

Comme pour l'évaluation de la fidélité, il y a tout intérêt à ce que la précision d'estimation d'une méthode de routine soit faite par plusieurs laboratoires avec des échantillons d'origine différente.

\subsection{Définition}

\section{SENSIBILITE}

- Sensibilité (22) : "C'est, au voisinage d'une valeur donnée de la grandeur à mesurer, le quotient de l'accroissement de la grandeur observée par l'accroissement correspondant de la grandeur à mesurer".

En général, la sensibilité n'est pas une caractéristique très importante des méthodes de routine, notamment dans le cas des appareils d'analyse donnant directement des mesures de concentration.

\subsection{Détermination de la sensibilité}

Si $\Delta x$ est l'accroissement de la mesure donnée par la méthode de routine et $\Delta y$ l'accroissement correspondant de la grandeur à mesurer, donnée généralement par la méthode de référence, la sensibilité est exprimée par le rapport $\frac{\Delta x}{\Delta y}$. Souvent la sensibilité des méthodes de routine est liée à celle de l'instrument de rinesure.

Note: il ne faut pas confondre la sensibilité avec le SELIL DE SENSIBILITE OU LIMITE DE DETECTION. La limite de détection d'une méthode d'analyse est une caractéristique particulière qui est définie par la plus faible valeur de la grandeur à mesurer qui puisse être effectivement détectée par la méthode ou l'appareil.

\section{CONCLUSION}

En raison de l'importance technique et économique des méthodes d'analyse de routine, qui sont utilisées dans des domaines aussi variés que le contrôle de la qualité des matières premières, le contrôle des fabrications, la sélection génétique, etc., il est indispen-

(21) A la limite, lorsqu'on analyse une série d'échantillons constitués par le même lait auquel on a enlevé ou additionné la même matière grasse, la précision d'estimation sera très élevée $\left(\sigma_{y, x}\right.$ sera voisin de zéro), c'est-à-dire que tous les points seront alignés.

(22) En anglais : sensitivity. 
sable que les paramètres donnant la valeur analytique des méthodes soient non seulement bien définis mais également qu'ils soient estimés selon des normes précises.

Ceci devrait permettre :

$1^{\circ}$ de pouvoir plus facilement comparer entre eux les travaux effectués par les différents laboratoires chargés d'étudier les nouvelles méthodes d'analyse,

$2^{\circ}$ de donner aux utilisateurs, dans un langage clair et précis et sous une forme exploitable, les principales caractéristiques analytiques des méthodes qu'ils emploient,

$3^{\circ}$ de faciliter, pour l'analyse d'un produit donné, l'établissement de normes de précision qui soient respectées par les constructeurs de matériels d'analyse.

\section{R és u m é}

Cette étude donne les définitions et les méthodes statistiques de calcul des principaux paramètres définissant la valeur analytique d'une méthode d'analyse de routine : fidélité (répétabilité, reproductibilité) et justesse (exactitude de calibrage, précision d'estimation).

\section{Su m mary}

This study give the definitions and statical methods for the evaluation of the main paramaters defining the analytical value of a routine method of analysis : precision (repeatability, reproducibility) and accuracy of the mean (calibration accurcy, accuracy).

\section{Remerciements}

Nous remercions vivement M. Hermier (I.N.R.A., Département de Technologie des Produits Animaux) et M. Courtier (A.F.N.O.R.) pour les corrections apportées au manuscrit. 